\title{
DISTRIBUIÇÃO DE INÓCULO DE FUNGOS MICORRÍZICOS ARBUSCULARES PARA SISTEMAS AGROFLORESTAIS NA AGRICULTURA FAMILIAR
}

\author{
Priscila Batista Miranda ${ }^{1}$; Andrea Hentz de Mello² ${ }^{2}$ Fernanda Dias Pereira ${ }^{3}$; Rosana \\ Quaresma Maneschy ${ }^{4}$.
}

\author{
${ }^{1}$ Discente da Faculdade de Ciências Agrárias (FCAM) da Universidade Federal do Pará (UFPA), bolsista Pibic-Interior, \\ priscilinha.b.m@hotmail.com \\ ${ }^{2}$ Professora Adjunta II da FCAM/UFPA,coordenadora dos projetos Pibic Interior e FAPESPA, andreahentz@ufpa.br \\ ${ }^{3}$ Engenheira agrônoma e bolsista FAPESPA; ${ }^{4}$ Professora Adjunta II da FCAM/UFPA
}

\begin{abstract}
RESUMO: A maioria dos solos de terra firme da Amazônia apresentam acidez elevada e deficiência de nutrientes, o que impede o satisfatório crescimento e desenvolvimento das plantas. Como alternativa para a produção de mudas de alta qualidade para a região, tem-se dado destaque para a utilização de fungos micorrízicos arbusculares (FMAs), que promovem o melhor desenvolvimento das plantas em condições ambientais adversas. Diante disso, objetivou-se com este trabalho a produção inoculante de FMAs e distribuição para os agricultores interessados implantá-lo em seus sistemas de cultivo em projetos de assentamentos da região. O trabalho foi realizado na Faculdade de Ciências Agrárias de Marabá, na Universidade Federal do Pará e em projetos de assentamentos da região, onde foram coletadas amostras de solo em várias áreas de cultivo para a extração de esporos, que foram multiplicados em cultivo armadilha com Brachiaria brizantha, a fim de produzir inoculante para distribuir aos agricultores interessados na tecnologia. Foi verificada a ocorrência de fungos micorrízicos arbusculares nos diversos sistemas de cultivo dos assentamentos envolvidos e foram identificadas famílias de agricultores interessadas na utilização do inoculante. A multiplicação dos esporos para a produção de inoculante foi satisfatória, visto que foi produzido em quantidade suficiente para atender a demanda de 25 famílias interessadas, para as quais foram distribuídas 1830 doses de inoculantes, ficando evidente o grande interesse dos agricultores em melhorar seus sistemas de produção.
\end{abstract}

PALAVRAS-CHAVE: micorrização, inovação tecnológica, sistema de produção.

\section{DISTRIBUTION OF INOCULUM AMF AGROFORESTRY SYSTEMS FOR THE FAMILY} FARM

\begin{abstract}
Most upland soil of the Amazon has high acidity and nutrient deficiency, which prevents the satisfactory growth and development of plants. As an alternative to producing high quality seedlings for the region, has given prominence to the use of arbuscular mycorrhizal fungi (AMF), which promote better development of plants in adverse environmental conditions. Therefore, the objective was to produce and distribute MF inoculants for farmers interested in deploying it in their cropping systems in settlement projects in the region. The study was conducted at the Faculty of Agrarian Sciences of Maraba, in the Federal University of Pará and settlement projects in the region, where soil samples were collected in several areas of cultivation for the extraction of spores, which were multiplied in trap culture Brachiaria brizantha, in order to produce inoculum for distribution to farmers interested in technology. The occurrence of arbuscular mycorrhizal fungi in different cropping systems of the settlements involved were verified and identified farming families interested in using the inoculant. The multiplication of spores to produce inoculum was satisfactory, since it was produced in sufficient quantity to meet the demand of 25
\end{abstract}

Agroecossistemas, v. 3, n. 1, p. 45-51, 2011 
families involved, which were distributed to 1830 doses of inoculation, demonstrating the great interest of farmers in improving their systems production.

KEY-WORDS: mycorrhiza, technological innovation, production system.

\section{INTRODUÇÃO}

Uma das principais limitações para a agricultura na Amazônia é que a maioria dos solos de terra firme apresentam acidez elevada e deficiências generalizadas de nutrientes, o que impede o satisfatório crescimento e desenvolvimento das plantas (OLIVEIRA; MOREIRA; MOREIRA, 1997).

Diante disso, tem se pensado em alternativas de tecnologias inovadoras para a produção de mudas de alta qualidade, utilizando espécies de interesse econômico para a produção familiar da região. Uma delas é a produção de inóculo de fungos micorrízicos arbusculares (FMAs), que aumenta a resistência da planta ao ataque de patógenos do sistema radicular e auxilia na capacitação de absorção de água (MOREIRA; SIQUEIRA, 2006), apresentando grande potencial biotecnológico para a agricultura, já que os FMAs ocorrem naturalmente nos solos e raízes das plantas nesta região (HENTZ, 2007).

Sendo assim, o objetivo deste trabalho foi produzir inoculante de FMAs e identificação de agricultores interessados em implantar essa tecnologia em seus sistemas de cultivo em projetos de assentamentos da região.

\section{MATERIAL E MÉTODOS}

Este trabalho foi desenvolvido no Laboratório de Microbiologia do solo da Faculdade de Ciências Agrárias (FCAM) UFPA, no Projeto de Assentamento Araras, situado no Município de São João do Araguaia-PA, no PA Palmares II no Município de Parauapebas-PA, no PA Agroextrativista Praialta Piranheira, localizado em Nova Ipixuna-PA e no PA Belo Horizonte, em São João do Araguaia-PA.

O trabalho foi dividido em três etapas, onde a primeira consistiu na coleta de amostras de solo no período de 2007 a 2009, nos PAs Araras (áreas de monocultivo, de SAFs, de capoeira queimada, capoeira de corte e queima e capoeira de corte e queima em regeneração e área do quintal agroflorestal), Palmares (áreas de roça de mandioca e pastagem) e no Agroextrativista Praialta Piranheira (área de pasto jovem, área de cultivo consorciado e pasto degradado). Em cada área foram coletadas aleatoriamente 10 amostras simples de solo e raízes (profundidade de $10 \mathrm{~cm}$ ), que foram misturadas constituindo amostras compostas e encaminhadas Laboratório de Microbiologia do Solo da FCAM, para posterior extração de esporos, pela técnica de peneiramento úmido 
de (GERDMAN; NICOLSON, 1963), e centrifugação em água e sacarose a $40 \%$ de (JENKINS, 1964). Em seguida, os esporos foram preparados em lâminas e identificados segundo suas características morfológicas (SCHENCK; PÉREZ, 1987; INVAM, 2001). Para avaliação da colonização micorrízica, as raízes foram clarificadas e coradas, segundo a metodologia adaptada (KOSKE; GEMMA, 1989; GRACE; STRIBLEY, 1991).

A segunda etapa consistiu na multiplicação destes esporos extraídos do solo dos PAs e de esporos. A multiplicação foi feita em cultivo armadilha, com Brachiaria brizantha em 35 vasos de $1000 \mathrm{~g}$ de capacidade, contendo areia estéril como substrato, onde foram colocados de 10 a 15 esporos por vaso, que permaneceram em casa de vegetação por aproximadamente 150 dias. Após verificada a esporulação, foi feito o inoculante que constituiu de uma mistura de $5 \mathrm{~g}$ de areia e esporos, acondicionados em sacos plásticos de 7x15 cm de dimensão.

$\mathrm{O}$ delineamento experimental utilizado foi inteiramente casualizado. Os dados foram compilados e processados no software estatístico SISVAR, seguindo o teste Tukey, com médias comparadas a um nível de $5 \%$ de probabilidade (FURTADO, 2000).

E, na terceira e última etapa, foram realizadas a identificação dos agricultores interessados em utilizar o inoculante e suas culturas de interesse, por meio de visitas a campo, capacitação destes através de palestras sobre produção de mudas inoculadas e distribuição do inoculante para estes agricultores.

\section{RESULTADOS E DISCUSSÃO}

No Projeto de Assentamento Araras foi observada a presença de seis espécies de FMAs: Acaulospora escrobiculata, Gigaspora margarita, Glomus clarum, Glomus etunicatum, Scutellospora heterogama e uma espécie com taxonomia não identificada. Com maior frequência de ocorrência do gênero Glomus e Acaulospora (Tabela 1).

Comparando as áreas de cultivo do PA Araras, o SAFs obteve maior número de esporos da espécie Acaulospora escrobiculata, Gigaspora margarita, Glomus etunicatum e Scutellospora heterogama, totalizando 63 (Tabela 1). De acordo com Loss et al. (2009), os sistemas agroflorestais podem manter ou aumentar a produtividade de determinado local, devido a processos que aumentam a entrada ou reduzem perdas no solo, como matéria orgânica, nutrientes e água, além de melhorar as propriedades físicas e químicas e beneficiar processos microbiológicos do solo, propiciando assim, um maior número de esporos de FMAs. 
Tabela 1. Número de esporos e frequência (FO \%) de FMAs em $50 \mathrm{~mL}$ das amostras de solos coletadas no Projeto de Assentamento Araras, no Município de São João do Araguaia - PA.

\begin{tabular}{lcccccc}
\hline Espécies & Cupuaçu & SAFs & $\begin{array}{c}\text { Capoeira } \\
\text { queimada }\end{array}$ & $\begin{array}{c}\text { Roça de } \\
\text { corte e queima }\end{array}$ & $\begin{array}{c}\text { Roça de } \\
\text { corte e queima } \\
\text { em regeneração }\end{array}$ & FO (\%) \\
\hline Acaulospora escrobiculata & $12 \mathrm{a}$ & $22 \mathrm{a}$ & $0 \mathrm{~d}$ & $0 \mathrm{~d}$ & $3 \mathrm{c}$ & 4 \\
Gigaspora margarita & $0 \mathrm{c}$ & $8 \mathrm{c}$ & $0 \mathrm{~d}$ & $0 \mathrm{~d}$ & $0 \mathrm{~d}$ & 1 \\
Glomus clarum & $0 \mathrm{c}$ & $0 \mathrm{~d}$ & $11 \mathrm{~b}$ & $11 \mathrm{~b}$ & $20 \mathrm{a}$ & 4 \\
Glomus etunicatum & $4 \mathrm{~b}$ & $21 \mathrm{a}$ & $22 \mathrm{a}$ & $22 \mathrm{a}$ & $6 \mathrm{~b}$ & 8 \\
Scutellospora heterogama & $4 \mathrm{~b}$ & $12 \mathrm{~b}$ & $1 \mathrm{c}$ & $1 \mathrm{c}$ & $2 \mathrm{c}$ & 2 \\
Não identificada & $0 \mathrm{~d}$ & $0 \mathrm{~d}$ & $0 \mathrm{~d}$ & $0 \mathrm{~d}$ & $1 \mathrm{~d}$ & 0,1 \\
\hline
\end{tabular}

Médias seguidas da mesma letra não diferem estatisticamente pelo método de Tukey (5\%) de probabilidade.

No quintal agroflorestal do PA Araras, foram encontradas cinco espécies de FMAs, das quais uma não foi identificada taxonomicamente, com maior ocorrência de
Glomus etunicatum e Glomus clarum, seguidos da espécie não identificada, com menor ocorrência dos gêneros Gigaspora e Scutellospora (Tabela 2).

Tabela 2. Número de esporos e frequência (FO \%) de FMAs em $50 \mathrm{~mL}$ das amostras de solos coletadas no Quintal Agroflorestal Projeto de Assentamento Araras, no município de São João do Araguaia - PA.

\begin{tabular}{lccccc}
\hline Culturas & $\begin{array}{c}\text { Gigaspora } \\
\text { margarita }\end{array}$ & $\begin{array}{c}\text { Glomus } \\
\text { clarum }\end{array}$ & $\begin{array}{c}\text { Glomus } \\
\text { etunicatum }\end{array}$ & $\begin{array}{c}\text { Scutellospora } \\
\text { heterogama }\end{array}$ & $\begin{array}{c}\text { Não } \\
\text { identificada }\end{array}$ \\
\hline Abacate & $0 \mathrm{c}$ & $2 \mathrm{c}$ & $5 \mathrm{~b}$ & $1 \mathrm{~b}$ & $0 \mathrm{~b}$ \\
Açaí & $0 \mathrm{c}$ & $8 \mathrm{a}$ & $4 \mathrm{~b}$ & $0 \mathrm{c}$ & $0 \mathrm{~b}$ \\
Banana & $0 \mathrm{c}$ & $3 \mathrm{c}$ & $2 \mathrm{c}$ & $1 \mathrm{~b}$ & $0 \mathrm{~b}$ \\
Cacau do mato & $0 \mathrm{c}$ & $0 \mathrm{~d}$ & $1 \mathrm{~d}$ & $0 \mathrm{c}$ & $1 \mathrm{~b}$ \\
Cajá & $4 \mathrm{a}$ & $6 \mathrm{~b}$ & $8 \mathrm{a}$ & $0 \mathrm{c}$ & $0 \mathrm{~b}$ \\
Carambola & $0 \mathrm{c}$ & $0 \mathrm{~d}$ & $0 \mathrm{~d}$ & $0 \mathrm{c}$ & $0 \mathrm{~b}$ \\
Cupuaçu nativo & $1 \mathrm{~b}$ & $0 \mathrm{~d}$ & $0 \mathrm{~d}$ & $1 \mathrm{~b}$ & $0 \mathrm{~b}$ \\
Cupuaçu & $0 \mathrm{c}$ & $0 \mathrm{~d}$ & $0 \mathrm{~d}$ & $0 \mathrm{c}$ & $3 \mathrm{a}$ \\
Manga & $0 \mathrm{c}$ & $0 \mathrm{~d}$ & $0 \mathrm{~d}$ & $0 \mathrm{c}$ & $3 \mathrm{a}$ \\
Pitanga & $0 \mathrm{c}$ & $1 \mathrm{~d}$ & $3 \mathrm{c}$ & $0 \mathrm{c}$ & $0 \mathrm{~b}$ \\
Pitomba & $0 \mathrm{c}$ & $0 \mathrm{~d}$ & $1 \mathrm{~d}$ & $0 \mathrm{c}$ & $0 \mathrm{~b}$ \\
Embaúba & $0 \mathrm{c}$ & $5 \mathrm{~b}$ & $0 \mathrm{~d}$ & $3 \mathrm{a}$ & $0 \mathrm{~b}$ \\
Mata & $0 \mathrm{c}$ & $0 \mathrm{~d}$ & $4 \mathrm{~b}$ & $0 \mathrm{c}$ & $0 \mathrm{~b}$ \\
\hline FO $(\%)$ & 0,5 & 2,5 & 2,8 & 0,6 & 0,7 \\
\hline
\end{tabular}

Médias seguidas da mesma letra não diferem estatisticamente pelo método de Tukey (5\%) de probabilidade. 
No quintal agroflorestal a cultura do cajá foi a que apresentou maior número de esporos, totalizando 18 esporos, pertencentes aos gêneros Gigaspora e Glomus (Tabela 2). Isso se explica pelo fato de que as maiorias das espécies arbustivas e arbóreas tropicais, frutíferas e florestais, com micorrizas, desenvolvem-se melhor e mais rapidamente (MIRANDA, 2006). No PA Palmares foram caracterizadas sete espécies de FMAs na roça de mandioca e no sistema de pasto. Com maior frequência do gênero Glomus e menor frequência de Acaulospora, seguida da espécie não identificada (Tabela 3).

Tabela 3. Número de esporos e frequência (FO \%) de FMAs em $50 \mathrm{~mL}$ das amostras de solos coletadas no PA Palmares, no Município de Parauapebas- PA.

\begin{tabular}{lccc}
\hline Espécies & Roça de mandioca & $\begin{array}{c}\text { Pastagem de braquiaria } \\
\text { (Brachiaria brizantha })\end{array}$ & FO $(\%)$ \\
\hline Acaulospora scrobiculata & $0 \mathrm{~d}$ & $1 \mathrm{c}$ & 0,1 \\
Gigaspora margarita & $0 \mathrm{~d}$ & $2 \mathrm{c}$ & 0,2 \\
Glomus clarum & $15 \mathrm{c}$ & $7 \mathrm{~b}$ & 2,2 \\
Glomus etunicatum & $20 \mathrm{~b}$ & $49 \mathrm{a}$ & 6,9 \\
Glomus manihots & $23 \mathrm{a}$ & $0 \mathrm{~d}$ & 2,3 \\
Scutellospora heterogama & $1 \mathrm{~d}$ & $1 \mathrm{c}$ & 0,2 \\
Não identificada & $0 \mathrm{~d}$ & $1 \mathrm{c}$ & 0,1 \\
\hline
\end{tabular}

Médias seguidas da mesma letra não diferem estatisticamente pelo método de Tukey (5\%) de probabilidade.

O número de esporos na pastagem com Brachiaria brizantha foi maior que na roça de mandioca, isso pode ser explicado porque as gramíneas do tipo Brachiaria spp, são colonizadas pela maioria dos fungos micorrízicos arbusculares. Além disso, a maior parte das pastagens na Amazônia é degradada, favorecendo, portanto, a colonização micorrízica.

No PA Agroextrativista Praialta Piranheira, nos diferentes sistemas de cultivos, pôde-se verificar uma diversidade de espécies de FMAs. Onde na área de cultivo, na área de pasto e jovem e de pasto degradado foram identificadas as mesmas espécies de esporos de FMAs: Acaulospora scr obiculata, Gigaspora margarita, Glomus clarum, Glomus etunicatum e Scutellospora heterogama, demostrando que a sustentabilidade destes solos pode ser garantida em decorrência das atividades microbianas, que desempenham papel importante na conservação e recuperação de áreas degradadas.

No PA Belo Horizonte foram identificadas sete famílias interessadas em utilizar o inoculante em seus sistemas de cultivo, com espécies de laranja (Citrus $x$ sinensis), leucena (Leucena leucocephala) e limão (Citrus $x$ limon.), totalizando 1080 
inóculos distribuídos. No PA Palmares II foram verificadas 18 famílias interessadas, as quais foram atendidas com 750 inoculantes para serem utilizados em cultivos de açaí (Euterpe oleracea), castanha do Brasil (Bertholletia excelsa), ipê roxo (Tabebuia avellanedae) e ipê amarelo (Tabebuia chrysotricha), que são de interesse destas famílias.

A multiplicação dos fungos foi realizada de forma satisfatória, visto que houve a produção de inoculantes suficiente para atender a demanda identificada nos assentamentos, onde foram distribuídos mil oitocentos e trinta (1830) doses de inoculantes, sendo cada dose composta de $3 \mathrm{~g}$.

\section{CONCLUSÕES}

Houve um grande interesse dos agricultores em melhorarem seus sistemas de cultivos, e este trabalho de pesquisa e extensão possibilitou difusão de tecnologia inovadora que proporciona melhores condições de nutrição às plantas, corroborando para sustentabilidade dos sistemas de produção familiares.

\section{REFERÊNCIAS}

FURTADO, D. F. Sistemas de análise estatística para dados balanceados. Lavras: UFLA/DEX/SISVAR, 2000. 145 p.

GRACE, C.; STRIBLEY, D. P. A safer procedure for roution staining of vesicular-ar mycorrhizal fungi. Mycological Research, v.95, n.10, p.1160-1162, 1991.

GERDEMAN, J. W.; NICOLSON, T. H. Spores of mycorrhizal Endogone species extracted from soil by wet and decanting. Trans. Br. Myc. Soc. v.46, p. 235-244, 1963.

HENTZ, A. N. Ocorrência, caracterização e eficiência de fungos micorrízicos arbusculares na Amazônia: uma alternativa para o cultivo sustentável. Apostila teórica apresentado à Faculdade de Ciências Agrárias da Universidade Federal do Pará, Marabá, 2007. 34 p.

INVAM. INTERNATIONAL CULTURE COLLECTION OF VESICULAR ARBUSCULAR MYCORRHIZAL FUNGI. 2001. Disponível em: $<$ http://invam.caf.wvu.edu/mycinfo/methods/c ultures/monosp.htm>. Acesso em: out. 2010.

JENKINS, W. R. Arapid centrifugal-floration technique for separating nematodes from soil. Plant Disease Report, v.48, 692 p.,1964.

KOSKE, R. E.; GEMMA, J. N. A modified procedure for roots to detect VA mycorrhizas. Mycological Research, v. 92, n. 4, p.458488, 1989.

LOSS, A.; ANGELINI, G. A. R.; PEREIRA, A. C. C.; LÃ, O. R.; MAGALHÃES, M. O. L.; SILVA, E. M. R. da. SAGGIN-JUNIOR, O. J. Atributos químicos do solo e ocorrência de fungos micorrízicos sob áreas de pastagem e sistema agroflorestal, 
Brasil. Seropédica: Embrapa Agrobiologia, 2009. 45 p.

MIRANDA, J. C. C. Porque a micorriza é importante para a produção agrícola, frutífera e florestal, 2006. Disponível em: < http://www.agrosoft.org.br/agropag/20721.ht m >. Acesso em: 14 mai. 2010.

MOREIRA, F. S.; SIQUEIRA, J. O. Microbiologia e Bioquímica do solo. ed. 2. Lavras: Ed. UFLA, 2006. 729 p.

OLIVEIRA, L. A.; MOREIRA, F. M. S.; MOREIRA, F. W. Ocorrências de microrganismos benéficos e ecossistemas amazônicos. In: NODA, H.; SOUZA, L. A.
G.; FONSECA, O. J. M. (Eds.) Duas décadas de contribuições do INPA à pesquisa agronômica no trópico úmido. Manaus: INPA, 1997. p. 221-240.

SCHENCK, N.; PERÉZ, Y. Manual for the identification of VA mycorrhizal fungi. 3.ed. Gainesville: Synbergistic Publications, 1987. $286 \mathrm{p}$. 